\title{
Feedforward attractor targeting for non- linear oscillators using a dual-frequency driving technique
}

Cite as: Chaos 30, 073123 (2020); https://doi.org/10.1063/5.0005424

Submitted: 02 March 2020 . Accepted: 15 June 2020 . Published Online: 14 July 2020

F. Hegedús (D), P. Krähling, M. Aron (D), W. Lauterborn (D), R. Mettin, and U. Parlitz (i)
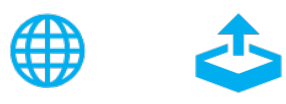

\section{ARTICLES YOU MAY BE INTERESTED IN}

Terminating transient chaos in spatially extended systems

Chaos: An Interdisciplinary Journal of Nonlinear Science 30, 051108 (2020); https://

doi.org/10.1063/5.0011506

Identifying edges that facilitate the generation of extreme events in networked dynamical systems

Chaos: An Interdisciplinary Journal of Nonlinear Science 30, 073113 (2020); https:// doi.org/10.1063/5.0002743

Asymptotic estimates of SARS-CoV-2 infection counts and their sensitivity to stochastic perturbation

Chaos: An Interdisciplinary Journal of Nonlinear Science 30, 051107 (2020); https:// doi.org/10.1063/5.0008834

\section{Sign up for topic alerts} New articles delivered to your inbox 


\title{
Feedforward attractor targeting for non-linear oscillators using a dual-frequency driving technique
}

\author{
Cite as: Chaos 30, 073123 (2020); doi: 10.1063/5.0005424 \\ Submitted: 2 March 2020 . Accepted: 15 June 2020 . \\ Published Online: 14 July 2020

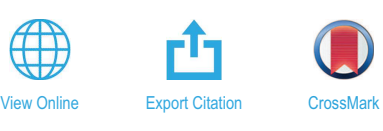

F. Hegedűs, , , a) (D) P. Krähling, ${ }^{1, b)}$ M. Aron, ${ }^{2, c)}$ (D) W. Lauterborn, ${ }^{3, d)}$ (D) R. Mettin, ${ }^{3, e)}$ and U. Parlitz $^{2, f)}$ (iD

\begin{abstract}
AFFILIATIONS
${ }^{1}$ Department of Hydrodynamic Systems, Faculty of Mechanical Engineering, Budapest University of Technology and Economics, Müegyetem rakpart 3, H-1111 Budapest, Hungary

${ }^{2}$ Research Group Biomedical Physics, Max Planck Institute for Dynamics and Self-Organization, Am Fassberg 17,

D-37077 Göttingen, Germany and Institut für Dynamik komplexer Systeme, Georg-August-Universität Göttingen, Friedrich-Hund-Platz 1, D-37077 Göttingen, Germany

${ }^{3}$ Drittes Physikalisches Institut, Georg-August-Universität Göttingen, Friedrich-Hund Platz 1, D-37077 Göttingen, Germany
\end{abstract}

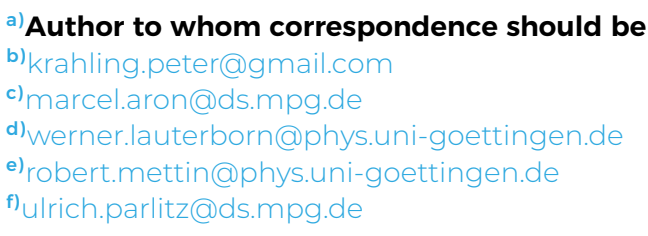

\section{ABSTRACT}

A feedforward control technique is presented to steer a harmonically driven, non-linear system between attractors in the frequency-amplitude parameter plane of the excitation. The basis of the technique is the temporary addition of a second harmonic component to the driving. To illustrate this approach, it is applied to the Keller-Miksis equation describing the radial dynamics of a single spherical gas bubble placed in an infinite domain of liquid. This model is a second-order, non-linear ordinary differential equation, a non-linear oscillator. With a proper selection of the frequency ratio of the temporary dual-frequency driving and with the appropriate tuning of the excitation amplitudes, the trajectory of the system can be smoothly transformed between specific attractors; for instance, between period- 3 and period- 5 orbits. The transformation possibilities are discussed and summarized for attractors originating from the subharmonic resonances and the equilibrium state (absence of external driving) of the system.

Published under license by AIP Publishing. https://doi.org/10.1063/5.0005424

The governing equations of many physical phenomena are nonlinear. One of the main consequences of non-linearity is the existence of various types of stable solutions (e.g., equilibrium, periodic, quasiperiodic, or chaotic) over the parameter space of the system. At a given parameter combination, many such stable states might co-exist exhibiting multi-stability. From an application point of view, the features mentioned above can cause unpredictable behavior as the different stable states usually represent different system performances. Therefore, it is mandatory to be able to drive a system to a desirable state. The control technique presented in this paper performs this task for periodic solutions of harmonically excited non-linear oscillators. The main strength of the method is that direct attractor selection is possible without the application of feedback on the system. Although the control procedure is demonstrated only for a specific system (Keller-Miksis equation), the generalization of the principles of the technique for other non-linear systems can significantly broaden its applicability to a large number of scientific fields in the future, for instance, in mechanical engineering, chemistry, laser physics, fluid dynamics, or social sciences, to name a few.

\section{INTRODUCTION}

In the parameter space of many non-linear systems, different domains of attractors occur, ${ }^{1-12}$ as illustrated in Fig. 1. These domains can overlap in certain parameter regions representing 


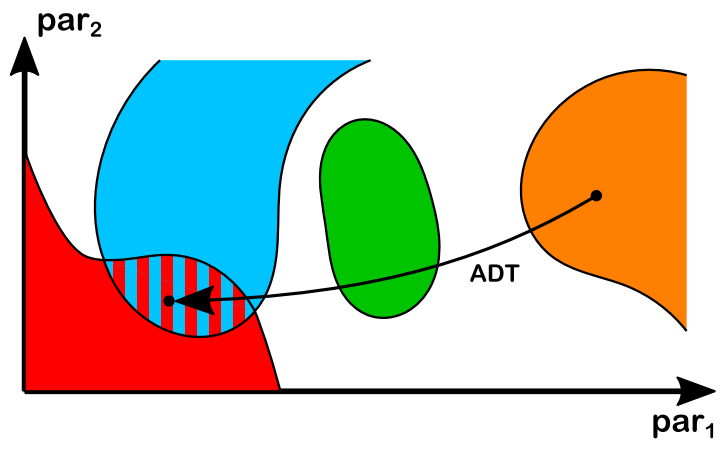

FIG. 1. Sketch of domains of attractors in a two-dimensional parameter space. The different colors represent different families of continuously connected attractors. The abbreviation ADT stands for the Attractor Domain Targeting technique.

multi-stability. The behavior of the system is usually different from domain to domain. For instance, chemical reactions can have different chemical yields for different attractors. ${ }^{1,11,13}$ In cardiac models, the different stable states can represent normal cardiac rhythm or lethal arrhythmia. ${ }^{14-17}$ In the case of a large number of coupled systems (e.g., power grids ${ }^{18}$ ), two stable states may display the difference between synchronous and asynchronous behavior. ${ }^{19-22}$ Therefore, the ability to drive a system between domains of stable states is important to maintain and control its performance. As the domains do not necessarily overlap each other, this is an extended concept of control of multi-stability. ${ }^{23-26}$ The proposed method that is capable of driving a system smoothly between stable states having distinct parameter sets is called Attractor Domain Targeting (ADT) technique throughout the present paper. The arrow between the two black dots in Fig. 1 represents this method. Note that this specific problem is a non-trivial task as the attractors represented by the two black dots may not be connected by a continuous transformation; in addition, in this example, the "blue" attractor may co-exist with the "red" one.

The main aim of this study is to present an ADT technique that works for harmonically driven non-linear oscillators. It is capable of smoothly steering such a system between periodic domains of attractors in the amplitude-frequency parameter plane of the excitation by temporarily adding a second harmonic component to the driving. The test model to demonstrate this approach is the Keller-Miksis equation, ${ }^{27}$ which is a second-order ordinary differential equation describing the radial dynamics of a single spherical gas bubble placed in an infinite domain of liquid. ${ }^{28-31}$ Results show that with a proper selection of the frequency ratio of the temporary dual-frequency driving and with the proper tuning of the excitation amplitudes, smooth transformations in the extended parameter space exist between certain periodic attractors (e.g., between period5 and period- 3 orbits). The initial and final state of the system during the control process is a single-frequency driven operation having distinct frequency values. That is, in both states (initial and final), different driving amplitudes corresponding to the two frequencies are zero, but not both of them.

Previously, ${ }^{23}$ this control method has been demonstrated to provide a transition between a period- 2 and a period-3 orbit corresponding to the subharmonic resonances of order $1 / 2$ and $1 / 3$, respectively. Here, we show transformations between stable orbits of any subharmonic resonance of order $1 / n(n=2, \ldots, 9)$. Moreover, direct routes from period-1 orbits originating from the equilibrium of the system (in the absence of external driving) to orbits of subharmonics with order higher than $1 / 5$ (the period is higher than 5) were also discovered. The advantages and disadvantages of our novel approach and a brief summary of possible applications are provided in Sec. V.

\section{THE TEST MODEL}

The test model employed in the present study is the Keller-Miksis equation ${ }^{27,30}$ describing the radial dynamics of a single spherical gas bubble placed in an infinite domain of liquid. The second-order, ordinary differential equation (ODE) reads

$$
\begin{aligned}
& \left(1-\frac{\dot{R}}{c_{L}}\right) R \ddot{R}+\left(1-\frac{\dot{R}}{3 c_{L}}\right) \frac{3}{2} \dot{R}^{2} \\
& =\left(1+\frac{\dot{R}}{c_{L}}+\frac{R}{c_{L}} \frac{d}{d t}\right) \frac{\left(p_{L}-p_{\infty}(t)\right)}{\rho_{L}},
\end{aligned}
$$

where $R(t)$ is the time-dependent bubble radius, the dot stands for the derivative with respect to time $t$, and $c_{L}=1497.3 \mathrm{~m} / \mathrm{s}$ and $\rho_{L}=997.1 \mathrm{~kg} / \mathrm{m}^{3}$ are the speed of sound and the density of the liquid, respectively. The pressure $p_{L}$ is the pressure in the liquid at the interface (without the far field pressure). The pressure far away from the bubble, $p_{\infty}(t)$, represents the external driving of the system. It is composed of static and periodic components,

$$
p_{\infty}(t)=P_{\infty}+P_{A 1} \sin \left(\omega_{1} t\right)+P_{A 2} \sin \left(\omega_{2} t+\theta\right) .
$$

Here, $P_{\infty}=1$ bar is the ambient pressure. The periodic components have pressure amplitudes $P_{A 1}$ and $P_{A 2}$, angular frequencies $\omega_{1}=2 \pi f_{1}$ and $\omega_{2}=2 \pi f_{2}$, and a phase shift $\theta$ according to the general, dual-frequency case.

The relationship between the pressures inside and outside the bubble at its interface can be written as

$$
p_{G}+p_{V}=p_{L}+\frac{2 \sigma}{R}+4 \mu_{L} \frac{\dot{R}}{R},
$$

where the total pressure inside the bubble is the sum of the partial pressures of the non-condensable gas, $p_{G}$, and the vapor, $p_{V}=3166.8 \mathrm{~Pa}$. The surface tension is $\sigma=0.072 \mathrm{~N} / \mathrm{m}$ and the liquid dynamic viscosity is $\mu_{L}=8.902^{-4} \mathrm{~Pa}$ s. The gas inside the bubble obeys a simple polytropic relationship

$$
p_{G}=\left(P_{\infty}-p_{V}+\frac{2 \sigma}{R_{E}}\right)\left(\frac{R_{E}}{R}\right)^{3 \gamma},
$$

where the polytropic exponent is chosen as $\gamma=1.4$ (air, adiabatic behavior) and the equilibrium bubble radius is $R_{E}=10 \mu \mathrm{m}$. Throughout the paper, the following dimensionless variables are used:

$$
\begin{gathered}
\tau=\frac{\omega_{1}}{2 \pi} t, \\
y_{1}=\frac{R}{R_{E}},
\end{gathered}
$$




$$
y_{2}=\dot{R} \frac{2 \pi}{R_{E} \omega_{1}} .
$$

The dimensionless ODE of the system is summarized in the Appendix.

The angular frequencies $\omega_{1}$ and $\omega_{2}$ are normalized by the linear, undamped eigenfrequency,

$$
\omega_{0}=\sqrt{\frac{3 \gamma\left(P_{\infty}-p_{V}\right)}{\rho_{L} R_{E}^{2}}-\frac{2(3 \gamma-1) \sigma}{\rho_{L} R_{E}^{3}}},
$$

of the unexcited system defining the relative frequencies,

$$
\begin{aligned}
& \omega_{R 1}=\frac{\omega_{1}}{\omega_{0}}, \\
& \omega_{R 2}=\frac{\omega_{2}}{\omega_{0}} .
\end{aligned}
$$

With a bubble size of $R_{E}=10 \mu \mathrm{m}$ and with the other constants in (8), the eigenfrequency is $\omega_{0}=340 \mathrm{kHz}$.

\section{A. The parameter space studied}

The investigated parameter space in the case of singlefrequency driving $\left(P_{A 2}=0\right)$ is composed of the pressure amplitude $P_{A 1}$ and the relative frequency $\omega_{R 1}$. The main objective of the singlefrequency driven simulations is to reveal the subharmonic bifurcation structure of the system. This is done because the Attractor Domain Targeting (ADT) technique is demonstrated for periodic orbits originating from the subharmonic resonances; for details, see Sec. III. The computation strategy is to perform one-dimensional scans with an initial value problem (IVP) solver, identify the subharmonic bifurcation structure, detect their bifurcation points, and follow the path of these points in the pressure-amplitude-relativefrequency parameter plane by utilizing the boundary value problem (BVP) solver AUTO. ${ }^{33}$ These calculations have low computational resource requirements (only one-dimensional scans and a few codim-2 bifurcation curves).

For the dual-frequency computations, high-resolution biparametric plots are produced in the $\left(P_{A 1}, P_{A 2}\right)$ plane for several fixed relative frequency combinations using an IVP solver. For simplicity, the relative phase shift between the harmonic components is set to $\theta=0$. The resolution of the parameter space is always $801 \times 501$ with 10 randomized initial conditions. Thus, a single plot consists of approximately $4 \times 10^{6}$ of IVPs. Simulations are conducted at every possible frequency combination, where the relative frequencies are chosen from the following set of values:

$$
\omega_{R 1,2}=2,3,4, \ldots, 9 .
$$

Taking into account symmetry properties of the possible combinations, this means 28 high-resolution bi-parametric plots, and approximately $112 \times 10^{6}$ IVPs have been solved altogether.

\section{B. The GPU-accelerated initial value problem solver}

Due to the resource-intensive computations for the solution of the IVPs, an in-house code (written in C++ and CUDA C) is used that is capable of exploiting the high processing power of graphics processing units (GPUs). The program package is a modular and general-purpose solver; for implementation details, capabilities, and performance characteristics, see its website, ${ }^{34}$ manual, ${ }^{35}$ and GitHub repository. ${ }^{36}$ The employed device here is an Nvidia GeForce GTX Titan Black card with 1.707 TFLOPS double-precision peak performance. The overall computation time of the $112 \times 10^{6}$ IVPs was only $16.8 \mathrm{~h}$.

\section{The global Poincaré section of the single- and dual-frequency driving}

In the case of single-frequency excitation $\left(P_{A 2}=0\right)$, the external driving is a purely harmonic function. Substituting Eq. (5) into Eq. (2), the dimensionless angular frequency of the first harmonic component is always $2 \pi$. Thus, a suitable global Poincaré section can be easily defined by the period of the driving $T_{1}=1$ (in the dimensionless time co-ordinate $\tau$ ). That is, the trajectories are sampled at time instances $\tau_{n}=n(n=0,1,2, \ldots)$.

For dual-frequency driving, the external forcing is not purely harmonic. Again, substituting Eq. (5) into Eq. (2), the two dimensionless angular frequencies are $2 \pi$ and $2 \pi \omega_{2} / \omega_{1}$; here, $\omega_{2} / \omega_{1}$ $=\omega_{R 2} / \omega_{R 1}$ is the frequency ratio. The corresponding periods are $T_{1}=1$ (as in the case of the single-frequency driving) and $T_{2}=\omega_{R 1} / \omega_{R 2}$. Keep in mind that the relative phase shift between the harmonic components is set to $\theta=0$. The ratio of the employed frequency pairs is always rational, see Eq. (11); thus, the dualfrequency driving is still periodic. This period $T$, which is the smallest common multiple of $T_{1}$ and $T_{2}$, can be used for defining the global Poincaré section of the dual-frequency driven system. That is, here, the trajectories are sampled at time instances $\tau_{n}=n \cdot T$ $(n=0,1,2, \ldots)$. As an example, for $\omega_{R 1}=5$ and $\omega_{R 2}=3$, the periods of the dimensionless dual-frequency driving are $T_{1}=1$, $T_{2}=5 / 3$, and $T=5$.

It must be stressed that the limiting cases of dual-frequency simulations in the $\left(P_{A 1}, P_{A 2}\right)$ bi-parametric plane-when one of the pressure amplitudes $P_{A 1}$ or $P_{A 2}$ is zero-represent single-frequency driven systems. However, the global Poincaré section still corresponds to the period of $T$. The consequence is that the periodicity of the periodic orbits is interpreted differently. For instance, in the case of the above example, a period- 5 attractor (obtained by sampling with $T_{1}$ ) in a single-frequency driven simulation with $\omega_{R 1}=5$ $\left(P_{A 2}=0\right)$ becomes only a period-1 orbit when the dual-frequency Poincaré section (given by $T$ ) is applied because $T=5 T_{1}$.

\section{THE SUBHARMONICS OF SINGLE-FREQUENCY DRIVING}

As the proposed ADT technique is demonstrated for orbits related to the subharmonic resonances of single-frequency excitation, it is mandatory to explore the corresponding bifurcation structure. Therefore, first, one-dimensional parameter studies are performed, where the first component of the Poincaré section $\Pi\left(y_{1}\right)$ is plotted as a function of the pressure amplitude $P_{A 1}$ at fixed relative frequencies $\omega_{R 1}$. An example can be seen in Fig. 2 with $\omega_{R 1}=4$. The pressure amplitude $P_{A 1}$ is varied between 0 bar and 20 bar with 


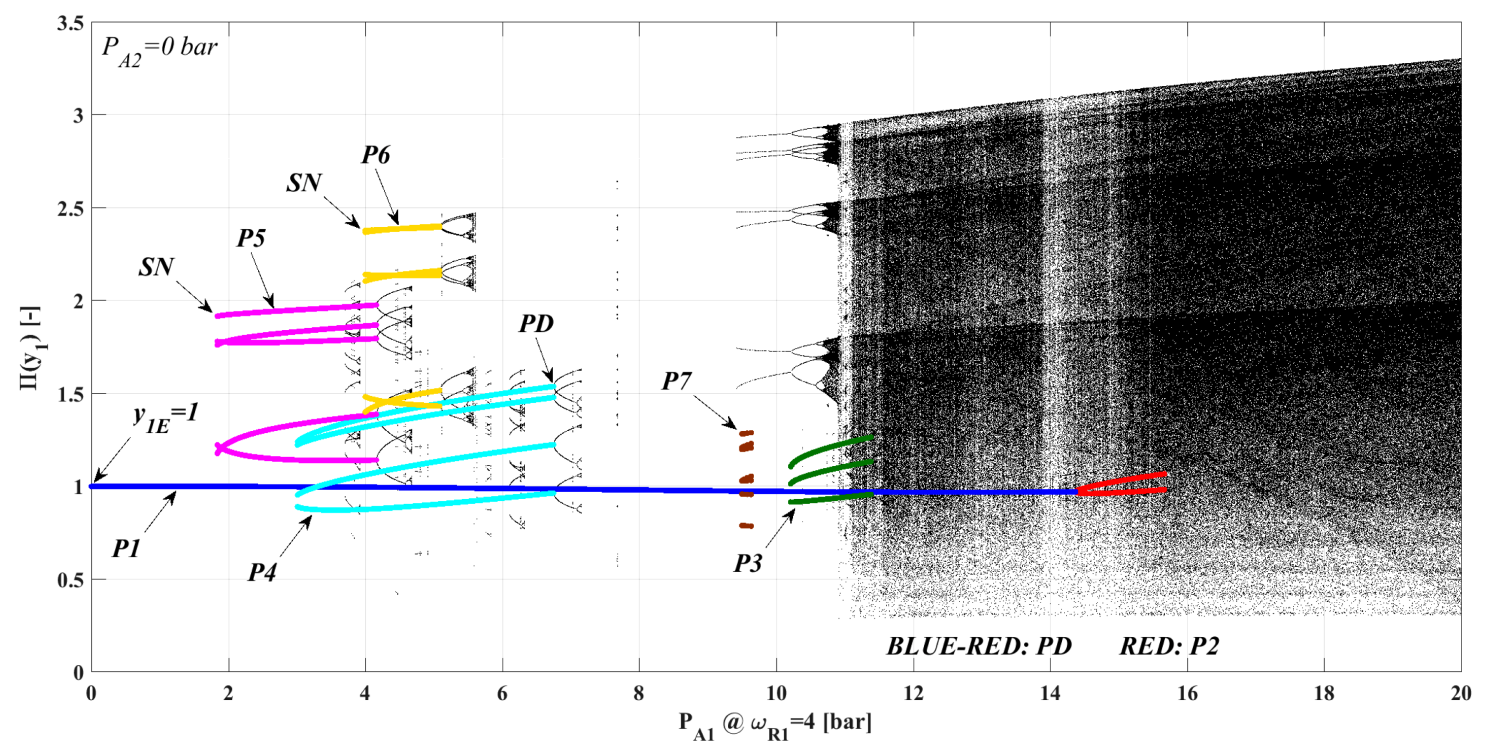

FIG. 2. One-dimensional bifurcation structure, where the first component of the Poincare section $\Pi\left(y_{1}\right)$ is presented as a function of the pressure amplitude $P_{A 1}$ at a fixed relative frequency $\omega_{R 1}=4$. The color-coded branches are the orbits (up to period-7) related to the subharmonic resonances of the order $1 / p$, where $p$ equals the period numbers $P 1, \ldots, P 7$ of the orbits. The dimensionless equilibrium (fixed point) of the system without driving $\left(P_{A 1}=0\right)$ is $y_{1 E}=1$. The labels $P D$ and $S N$ are the period-doubling and saddle-node bifurcation points, respectively.

an increment of 0.0004 bar. To reveal the co-existing attractors relevant for the ADT technique, five randomized initial conditions are used at every parameter value. The first 4096 iterations of the Poincaré map are regarded as initial transient and discarded. One iteration means the integration of the system by one cycle of the external driving $T_{1}$ (single-frequency driving). After the transient, subsequent 64 points in the Poincaré section are recorded and presented in Fig. 2.

Figure 2 shows a highly complex bifurcation structure, where several periodic and chaotic domains exist. Many of these domains

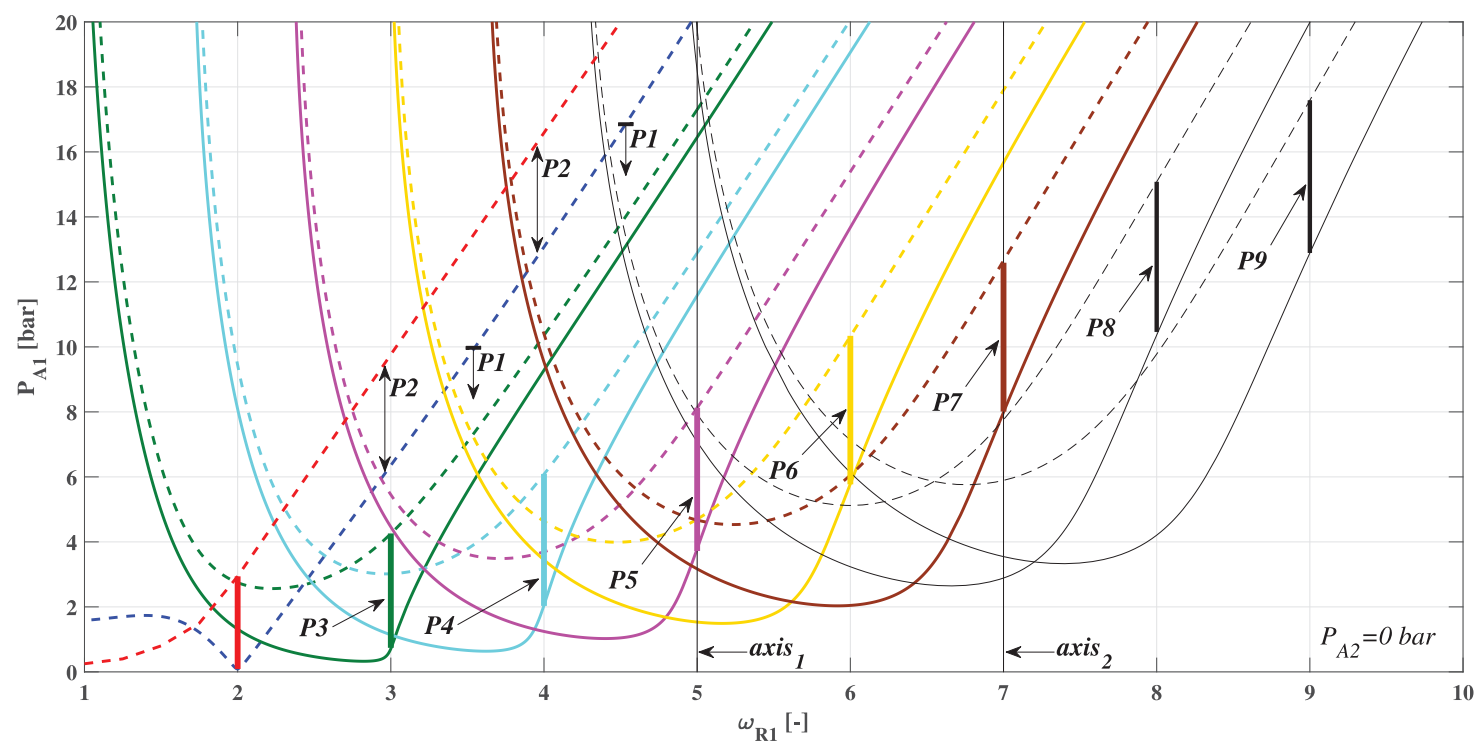

FIG. 3. The subharmonic resonance structure of the system. The color code is the same as in Fig. 2. The solid and dashed lines are saddle-node SN and period-doubling PD curves, respectively. With the Attractor Domain Targeting (ADT) technique, trajectories of the system can be guided between solutions lying on the thick vertical lines. 
overlap, exhibiting a high degree of multi-stability. This study focuses on the subharmonic resonances of order $(1 / p)$, where $p=2, \ldots, 9$ is the period number of the corresponding orbit. Up to period 7 , these structures are highlighted by the color-coded branches in Fig. 2; their period numbers are denoted as $P 2, \ldots, P 7$. The coloring of the period- 8 and period- 9 attractors is omitted for better visibility. The period-1 solutions (blue curve, $P 1$ ) originating from the equilibrium state of the bubble $y_{1 E}=1$ will be referred to in the subsequent discussion. The period-2 bifurcation curve (red) is born through a period-doubling PD point. All the other subharmonic branches appear via a saddle-node $S N$ bifurcation. The right end of each colored branch is a $P D$ point.

The bifurcation points of both sides of the color-coded periodic branches presented in Fig. 2 are tracked in the $\left(P_{A 1}, \omega_{R 1}\right)$ parameter plane. The results are shown in Fig. 3, where the color code is the same as in Fig. 2. Keep in mind that it is still a singlefrequency driven case. The solid and dashed lines are saddle-node $S N$ and period-doubling $P D$ bifurcation curves, respectively. Below the dashed dark blue curve (see also the arrows with $P 1$ ), period1 orbits exist. The area between the blue and red dashed curves is the region of period- 2 attractors. In the case of higher periods, the domains of stable states are bounded by a solid and a dashed curve with the same color. The arrows with the text mark the period numbers of the solutions. The bifurcation curves summarized in Fig. 3 are the subharmonic resonances of the system. Observe that the period- 8 and period- 9 subharmonics are shown as thin black lines (to avoid the overuse of different colors).

The periodic domains presented in Fig. 3 are not connected in the sense that tuning merely the excitation amplitude $P_{A 1}$ and the frequency $\omega_{R 1}$, the system cannot be driven arbitrarily from one domain to another. This is also shown in Fig. 2, where the majority of the color-coded branches are not connected (except the red and blue curves). Keep in mind that the different stable periodic domains in the $\left(P_{A 1}, \omega_{R 1}\right)$ plane can represent different system performances; see again Sec. I. Our technique-presented in Sec. IV in detail-is capable of guiding a trajectory between orbits occurring at parameter values corresponding to the thick vertical lines plotted in Fig. 3. Once the trajectory is on a specific attractor in a specific domain (e.g., on a period-6 solution between the yellow curves), it can be steered within this domain easily by tuning the parameters of the periodic driving. In this way, control of multi-stability can also be achieved in the case of overlapping periodic domains.

\section{THE DUAL-FREQUENCY TARGETING TECHNIQUE}

The basis of our Attractor Domain Targeting (ADT) technique to "transform" the system between attractors of different periods presented by the vertical lines in Fig. 3 is the temporary addition of a second harmonic component to the driving. This introduces an additional pair of control parameters: $P_{A 2}$ and $\omega_{R 2}$. The frequency ratio $\omega_{R 1} / \omega_{R 2}$ is fixed and must be equal to the ratio of the periods (or period numbers) of the attractors being transformed. For instance, for the transformation between the period-5 (P5) and period-6 (P6) orbits, $\omega_{R 1} / \omega_{R 2}=6 / 5$. Throughout this study, $\omega_{R 1}$ is always the higher relative frequency. Observe that the relative frequency values and the corresponding period numbers of the vertical lines in Fig. 3 coincide. Therefore, the relative frequency value of, e.g., $\omega_{R 1}=6$, is strongly related to the period-6 orbits on the thick yellow line. During the control process (tuning of the amplitudes $P_{A 1}$ and $P_{A 2}$ ), there is a dual-frequency driving, whereas, at the initial and end state, the system is driven by a single-frequency with distinct relative frequency values of $\omega_{R 1}$ or $\omega_{R 2}$, respectively. There is another kind of possible transformation: a direct route exists from a period-1 orbit (located below the blue dashed curve in Fig. 3) to one of the vertical lines if the relative frequency difference is $\omega_{R 1}-\omega_{R 2}=1$ and if $\omega_{R 1,2}>5$.

\section{A. Transformation between subharmonics}

In order to reveal the transformation possibilities between the subharmonic resonances (vertical lines in Fig. 3), high-resolution bi-parametric plots are created with the pressure amplitudes $P_{A 1}$ and $P_{A 2}$ as control parameters at several fixed relative frequency combinations. According to Eq. (11) and the related discussion, the total number of frequency combinations is 28 . Figure 4 shows four examples, where the $3 \mathrm{D}$ representation of the second component of the Poincaré section is plotted as a function of the control parameters. The resolutions of $P_{A 1}$ and $P_{A 2}$ are 801 and 501, respectively. Their ranges are adjusted according to the upper bound of the vertical lines in Fig. 3. To find the co-existing attractors, ten randomized initial conditions are applied.

The color-coded branches in Fig. 4 are periodic orbits using the period of the single-frequency excitation $T_{1}$ (if $P_{A 2}=0$ ) or $T_{2}$ (if $P_{A 1}=0$ ) for defining a global Poincaré section. The projections of these branches to their control parameter axes in Fig. 4 correspond to the thick vertical lines in Fig. 3. The arrows mark the period numbers of the solutions. The color-coding is the same as in Figs. 2 and 3. The dark and light gray points in Fig. 4 are the period-1 orbits using the period of the dual-frequency driving $T$ for defining a global Poincaré section. These solutions form surfaces connecting the colored branches (Fig. 4) in the single-frequency driven limit cases. Therefore, with the proper tuning of the pressure amplitudes $P_{A 1}$ and $P_{A 2}$, the system can be smoothly transformed through the gray surfaces between different periodic orbits of singlefrequency driving. It must be stressed that in these figures, three different interpretations are employed for the periodicity according to the definition of the Poincaré section; for the detailed discussion, the reader is referred back to Sec. II C. It is worth noting that the bifurcation structure of the dual-frequency driving is quite complex (see, e.g., Figs. 2 and 3 in our previous study ${ }^{23}$ ); therefore, only the period- 1 orbits are presented in Fig. 4 to remain focused and avoid overcrowded diagrams.

An example of the transformation between a period- 4 and a period- 6 attractor is demonstrated via an animation that can be found as multimedia. The last frame of the animation is presented in Fig. 5 (Multimedia view). The 3D panel on the left-hand side of the figure is the second component of the Poincaré section presented already in Fig. 4(c). Here, the red curve denotes the route of the transformation. The top panel on the right-hand side shows the dimensionless bubble wall velocity $y_{2}$ as a function of the dimensionless time $\tau$. The light blue, yellow, and black curves are the initial (period-4), the final (period-6), and the instantaneous solutions during the transformation, respectively. Since Fig. 5 (Multimedia 

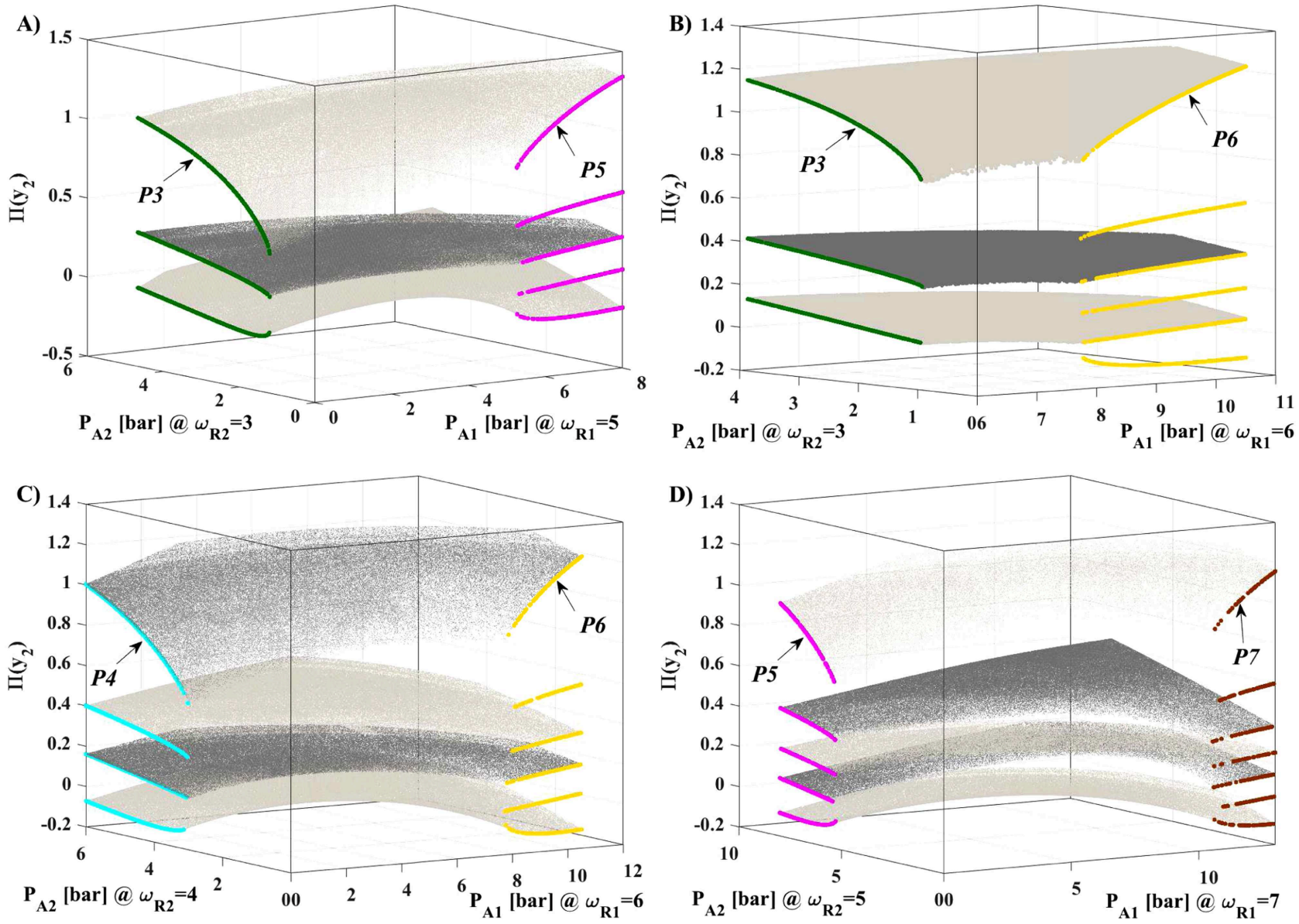

FIG. 4. Transformation surfaces between periodic orbits presented by the thick vertical lines in Fig. 3 via dual-frequency driving. The color-coded branches are periodic orbits of single-frequency driving (sampled with $T_{1}$ or $T_{2}$ ), where the labels $P 3, \ldots, P 7$ denote their period numbers. The dark and light gray points are period-1 attractors of the dual-frequency driving (sampled with $T$ ). Subpanels (a), (b), (c), and (d) are related to the frequency combinations of $\left(\omega_{R 1}, \omega_{R 2}\right)=(5,3),(6,3),(6,4)$, and $(7,5)$, respectively.

view) is the last frame of the animation, the yellow and black curves coincide. The bottom panel on the right-hand side is the signal of the external driving $P_{A}(\tau)$ (used here as a general notation for both single- and dual-frequency driving). The thin curve is related to the initial single-frequency driving ( $\omega_{R 2}=4$, period- 4 orbit), while the thick curve is the instantaneous driving signal of the dualfrequency transformation that also ends in a single-frequency driven case $\left(\omega_{R 1}=6\right.$, period- 6 orbit $)$. The values of the control parameters of the external driving $P_{A 1}, \omega_{R 1}, P_{A 2}$, and $\omega_{R 2}$ are depicted as bold texts in the top of the figure/animation. Note that only the pressure amplitudes change during the process. They are updated at every period of the dual-frequency driving $T=6$. The rate of change of the amplitudes has to be sufficiently small to remain within the basin of attraction of the solutions.

Although only four examples are shown in Fig. 4, smooth transformations are possible between any pair of periodic orbits. Keep in mind that the frequency ratio must be equal to the ratio of the periods (or period numbers) of the orbits being transformed. For instance, in Fig. 4(a), the period numbers are 5 and 3, and the relative frequencies are also $\omega_{R 1}=5$ and $\omega_{R 2}=3$, respectively. Note that not all branches are connected in general. In Fig. 4(a), all the green branches are connected to one of the purple branches, while there are purple branches that are not connected to a green branch. We call this phenomenon partial connectivity. In this sense, the transformation from period-3 to period-5 orbits is "safe," but the transformation in the opposite direction is "non-safe" (it depends on the initial branch defined by the relative phase in time of the solution). Naturally, there are period-1 surfaces initiated from these "orphan" branches; however, they are not presented here to avoid overcrowding of the figures. As a final remark, the dual-frequency diagrams can be regarded as connections between one-dimensional bifurcation structures at different frequencies. Compare the two vertical thin lines marked as axis $s_{1}$ and axis $_{2}$ in Fig. 3 with the dual-frequency case of Fig. 4(d). 

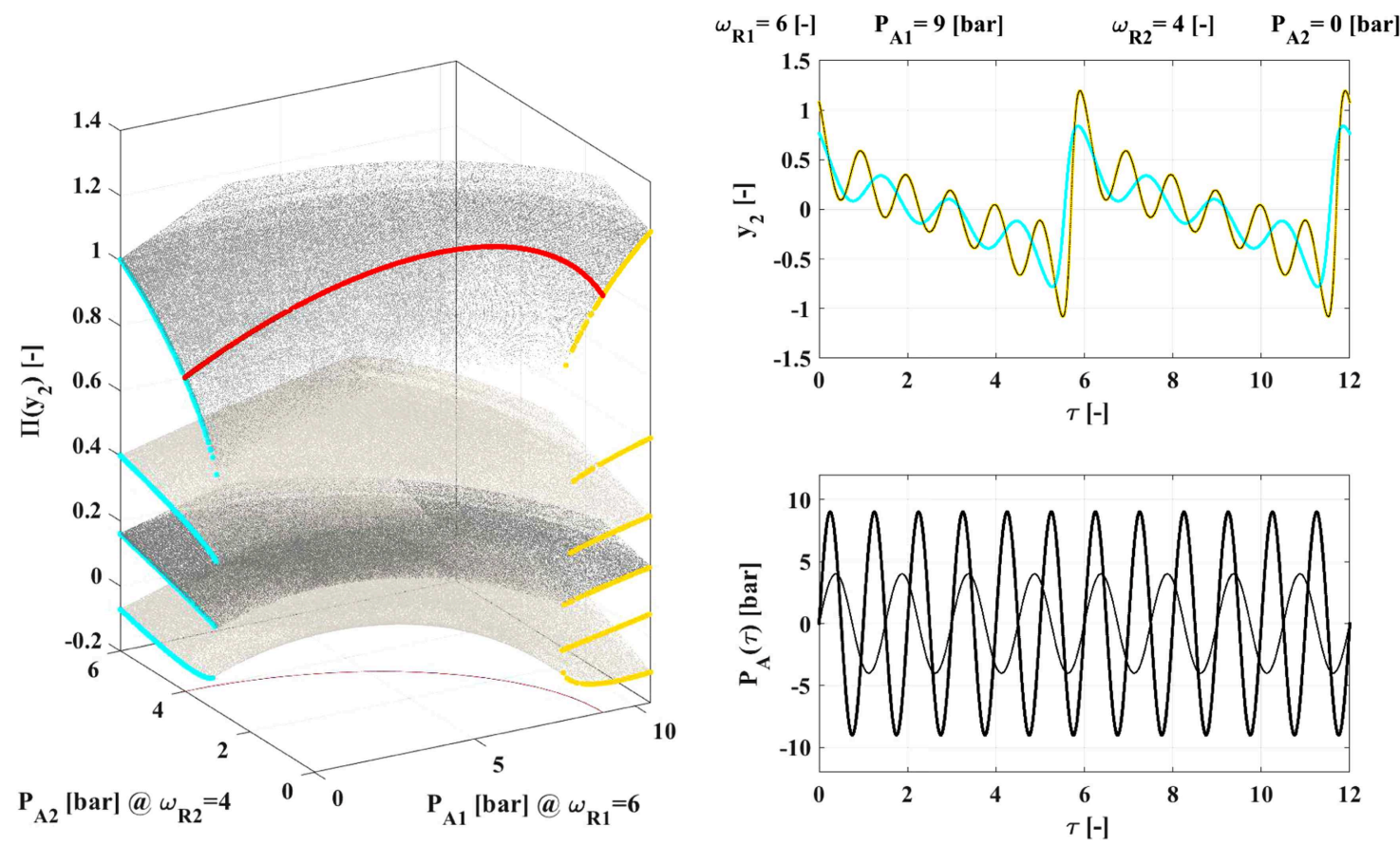

FIG. 5. The last frame of a transformation between a period-4 and a period-6 attractor via a temporary dual-frequency driving, see also Fig. 4(c). The red curves show the route of the transformation. The top panel on the right-hand side represents the time series of the dimensionless bubble wall velocities $y_{2}$, while the bottom panel on the right-hand side shows the signal of the external driving $P_{A}(\tau)$. Multimedia view: https://doi.org/10.1063/5.0005424.1

\section{B. Direct routes from period-1 orbits}

Another possibility to reach one of the subharmonic periodic domains (period-2 to period-9, vertical lines in Fig. 3) is the direct route from period- 1 orbits. These period- 1 solutions exist below the dashed dark blue codim-2 bifurcation curve shown in Fig. 3; see also the dark blue solid line in Fig. 2. An example for the direct route is presented in Fig. 6 produced in the same way as Fig. 4. The notations, the color-coding, the considerations for the Poincare sections, and the periodicities are also the same. The employed frequency combination is $\omega_{R 1}=6$ and $\omega_{R 2}=5$. Here, only a particular period-1 surface (gray) is presented. Its origin is the equilibrium solution $y_{2 E}=0$ of the unexcited system; its single-frequency limit cases are depicted by the period-1 (according to $T_{1}$ or $T_{2}$ ) blue lines. Interestingly, the surface tears apart, creating a hole near the middle and extending into an overlapping surface, where one of its sides is connected to a period-5 branch and the other one is attached to a period- 6 branch. Panels (a) and (b) of Fig. 6 show the same figure from different angles to visualize that the gray points form a single, overlapping surface. This example demonstrates the existence of a direct connection between the period-1 orbits and the subharmonics.

An example of a direct route transformation between a period1 and a period- 6 attractor is demonstrated via an animation that can be found as multimedia. The last frame of the animation is presented in Fig. 7 (Multimedia view). The structure of the figure is the same as in the case of Fig. 5 (Multimedia view). The 3D panel on the left-hand side is a copy of Fig. 6 in another perspective. The red curve denotes the route of the transformation. Observe that initial and end states of the transformation have the same (single frequency) parameter combination; thus, control of multi-stability is achieved in this specific example. In the top panel on the right-hand side, the blue, yellow, and black curves are the initial (period-1), final (period-6), and instantaneous solutions during the transformation, respectively. The yellow and black curves coincide since Fig. 7 (Multimedia view) is the last frame of the animation. The bottom panel on the righthand side is the signal of the external driving $P_{A}(\tau)$. There is only a single curve here as the initial and end states are related to the same single-frequency driving $\left(\omega_{R 1}=6\right)$. The values of the control parameters of the external driving $P_{A 1}, \omega_{R 1}, P_{A 2}$, and $\omega_{R 2}$ are depicted as bold texts in the top of the figure/animation. Note again that only the pressure amplitudes are changing during the steering process. Similarly, as in the case of the previous animation, attention has to be paid to the speed of change of the pressure amplitudes.

\section{DISCUSSION}

Table I summarizes the possible transformations between periodic attractors of all frequency combinations up to relative frequencies $\omega_{R 1,2}=9$. The rows and the columns are related to the high $\left(\omega_{R 1}\right)$ and low $\left(\omega_{R 2}\right)$ relative frequencies, respectively. Due to the normalization via Eqs. (9) and (10), the values of $\omega_{R 1}$ and $\omega_{R 2}$ are equal to the period numbers of the solutions being transformed. 

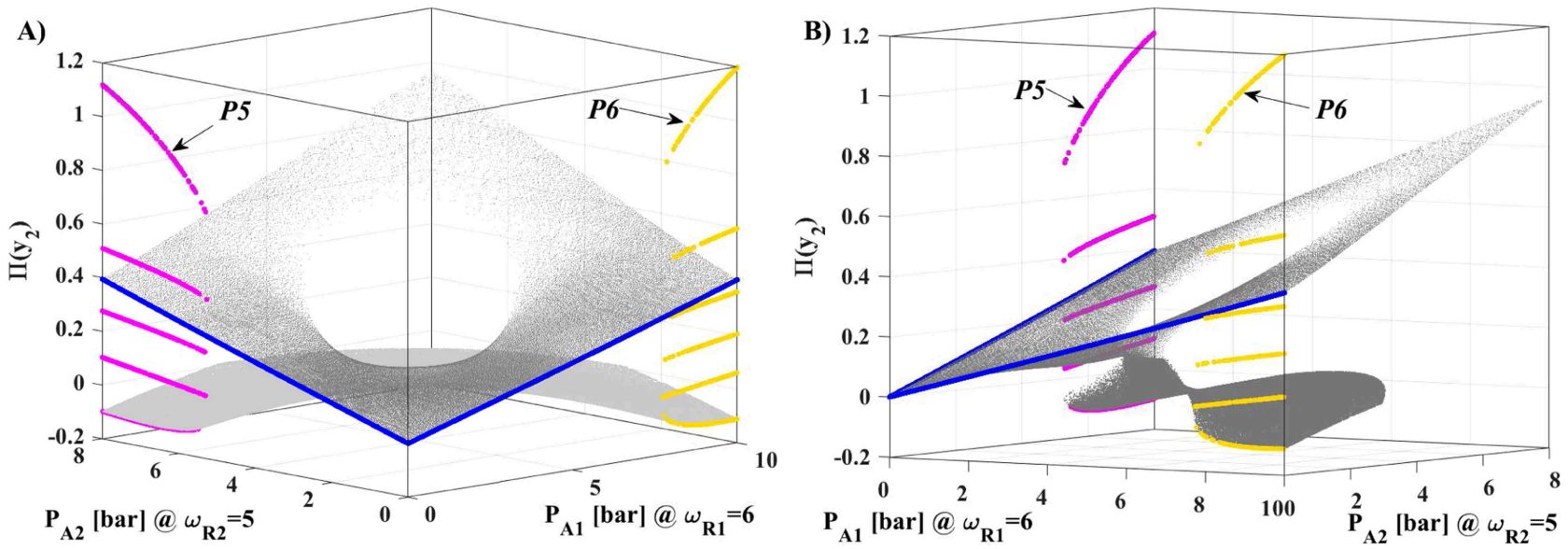

FIG. 6. Transformation between period-1 orbits and periodic attractors of the subharmonics. The color-coded branches are periodic orbits of single-frequency driving (sampled with $T_{1}$ or $T_{2}$ ), where $P 5$ and $P 6$ denote their period numbers. The gray points are period-1 attractors of the dual-frequency driving (sampled with $T$ ). Panels (a) and (b) show the same figure but from a different perspective to properly visualize the gray surface.

The upper half (due to the symmetry property) and the diagonal (ordinary single-frequency driving) of the table are excluded. The two numbers in each item of the table define the connectivity numbers $\left(c_{1}, c_{2}\right)$. The first number $c_{1}$ describes how many branches are connected from the attractor with the high period number (high frequency, $\left.\omega_{R 1}\right)$ to at least one branch of the solution with the low period number (low frequency, $\omega_{R 2}$ ). Since the relative frequencies are equal to the period numbers (also the number of the branches) of the solutions being transformed-see the period numbers of the vertical lines in Fig. 3 and their respective relative frequency values-the transformation from $\omega_{R 1}$ to $\omega_{R 2}$ is "safe" only when $c_{1}=\omega_{R 1}$. This condition is fulfilled only in one case discussed below in detail. The second number, $c_{2}$, describes connectivity in the opposite direction. Because $c_{2}=\omega_{R 2}$ in every entry of Table I, the transformation from a low to a high frequency is always "safe." That is, all the branches on the $\omega_{R 2}$ side are connected to at least one branch on the $\omega_{R 1}$ side. The label E denotes the transformation possibility directly from the period-1 attractor.

For the majority of the entries in Table I, without underlining, the two connectivity numbers are equal. From these, the bold-faced entries are the cases shown in Sec. IV in Figs. 4 and 6. Note how the numbers in the table agree with the number of gray surfaces in these figures. As stated before, these cases have partial connectivity; that is, the transformation is "safe" only in one direction (since $c_{1} \neq \omega_{R 1}$ ). The entries with underlining show frequency pairs having different connectivity numbers $\left(c_{1} \neq c_{2}\right)$. Among them, there is only one frequency combination having full connectivity: $\left(\omega_{R 1}, \omega_{R 2}\right)=(4,3)$ $=\left(c_{1}, c_{2}\right)$; see the sole bold-faced entry with underlining in Table I. In this case, all the four branches of the period- 4 orbits are connected to one of the branches of the period-3 solutions. This also means that there must be a branch of the period-3 orbits, which is connected to at least two branches of the period- 4 attractors. This example is demonstrated in Fig. 8(a). It can be seen that there is a surface that is torn apart and connects two branches from the period- 4 orbits to a single branch in its period- 3 side. This special surface is also shown in panel (b) from a different perspective for a better view. The other examples in Table I with underlining (but without bold-faced highlighting) have such a property that a single branch on one side is connected to multiple branches on the other side, e.g., frequency combinations $\left(\omega_{R 1}, \omega_{R 2}\right)=(5,2)$ or $\left(\omega_{R 1}, \omega_{R 2}\right)=(8,5)$. However, in these cases, the values of the frequency pairs are not equal to the values of the connectivity numbers $\left(\omega_{R 1}, \omega_{R 2}\right) \neq\left(c_{1}, c_{2}\right)$, which means partial connectivity.

In summary, from Table I, one can conclude that the transformation of the trajectories from low to high periodicity is always "safe." That is, regardless of the initial branch on the low period solutions, there always exists a surface that ends on the high period orbits. Observe that the second numbers in the table are always equal to the number in the first row (header line) of the same column. The transformation from high to low periodicity is safe only in the case of the relative frequency combination $\omega_{R 1}=4$ and $\omega_{R 2}=3$. Therefore, if one intends to drive the trajectory from a high period solution to a lower one, the pressure amplitude must be tuned down first to reach the period-1 orbit (see, e.g., Fig. 2), the relative frequency must be adjusted to $\omega_{R}=2$, and the pressure amplitude must be tuned up again to be on the period-2 bifurcation branch presented by the red vertical line in Fig. 3. From here, the transformation to high periodicities can be initiated that is always safe. Another option is to adjust the system to a period-1 orbit and use one of the direct routes (marked by $\mathrm{E}$ in Table I) to reach an orbit with periodicity higher than period 5 .

An alternative approach to the presented Attractor Domain Targeting (ADT) method to drive the system to a desired periodic orbit is to set up a proper pair of the pressure amplitude, $P_{A 1}$, and the relative frequency, $\omega_{R 1}$, according to Fig. 3 . Next, if the system is on an attractor different from the desired one, existing method $\mathrm{s}^{24}$ for control of multi-stability can be applied. In order to guarantee that the trajectory shall settle down to the desired orbit, the application 

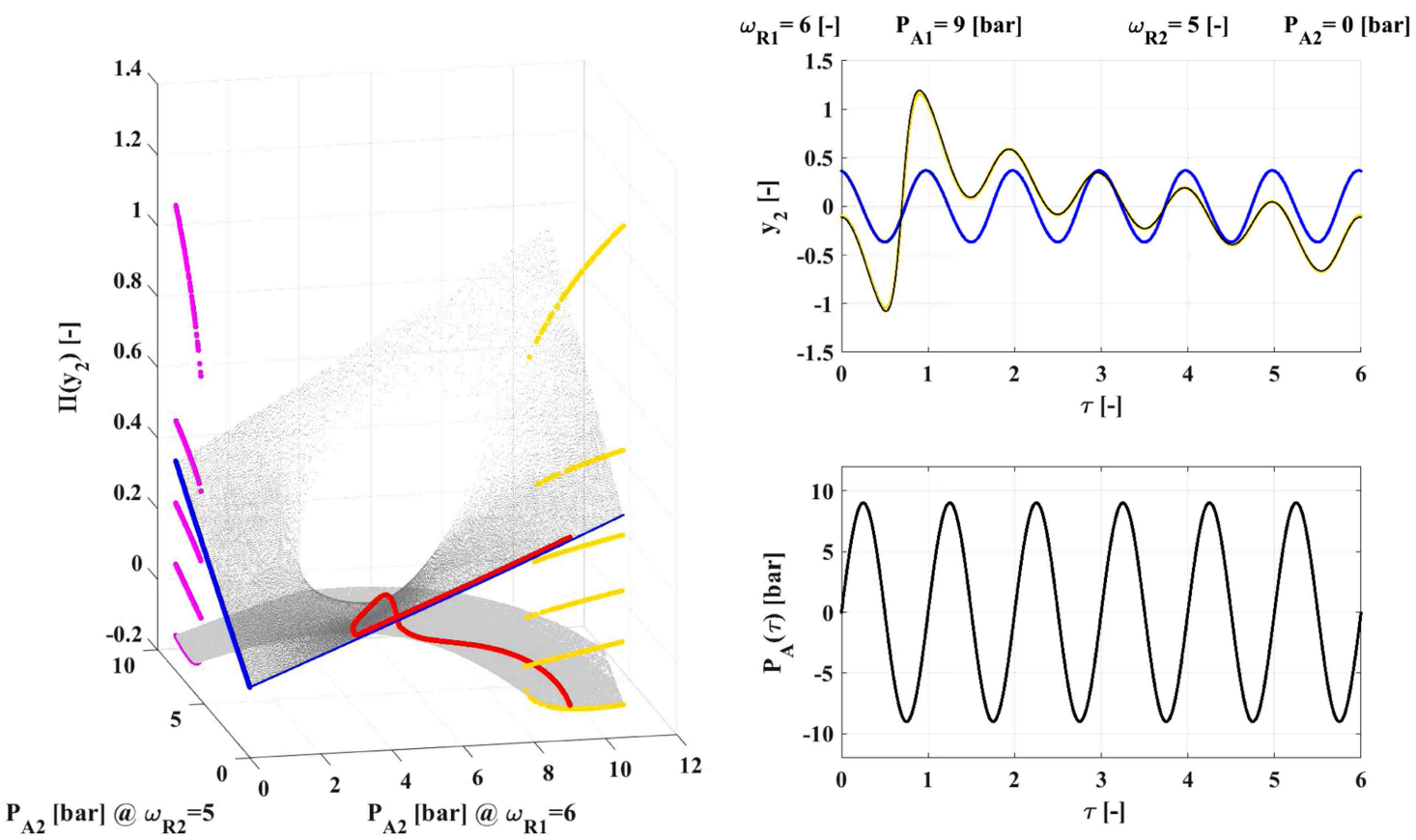

FIG. 7. The last frame of a transformation (a direct route) between a period-1 and a period-6 attractor via a temporary dual-frequency driving (see also Fig. 6). The red curve shows the route of the transformation. The top panel on the right-hand side represents the time series of the dimensionless bubble wall velocities $y_{2}$, while the bottom panel on the right-hand side shows the signal of the external driving $P_{A}(\tau)$. Multimedia view: https://doi.org/10.1063/5.0005424.2

of feedback control ${ }^{37-50}$ is mandatory. However, there are systems where the application of a feedback control is not possible (as in the case of acoustic bubble models ${ }^{30,51-61}$ ) due to the required continuous sampling of the solution in the state space or even the Jacobian of the system. The ADT technique is a non-feedback method $^{25,62-81}$ in the sense that no detailed knowledge on the current

TABLE I. Summary of the connectivity numbers $c_{1}$ and $c_{2}$ of the different periodic attractors as a function of the relative frequency combinations. The bold-faced entries without underlining are the cases presented in Sec. IV in Figs. 4 and 6. The bold-faced entry with underlining is the sole case with full connectivity shown in Fig. 8. In general, in the case of entries with underlining, the connectivity numbers are not equal $\left(c_{1} \neq c_{2}\right)$.

\begin{tabular}{|c|c|c|c|c|c|c|c|c|}
\hline & \multicolumn{7}{|c|}{$\omega_{R 2}$} \\
\hline & & 2 & 3 & 4 & 5 & 6 & 7 & 8 \\
\hline \multirow[t]{8}{*}{$\omega_{R 1}$} & 2 & $\ldots$ & $\ldots$ & $\ldots$ & $\ldots$ & $\cdots$ & $\cdots$ & $\ldots$ \\
\hline & 3 & 2,2 & $\ldots$ & $\ldots$ & $\ldots$ & $\ldots$ & $\ldots$ & $\ldots$ \\
\hline & 4 & 2,2 & 4,3 & $\ldots$ & $\ldots$ & $\ldots$ & $\ldots$ & $\ldots$ \\
\hline & 5 & 3,2 & $\overline{3,3}$ & 4,4 & $\ldots$ & $\ldots$ & $\ldots$ & $\ldots$ \\
\hline & 6 & 2,2 & 3,3 & 4,4 & $5,5, \mathrm{E}$ & $\ldots$ & $\ldots$ & $\ldots$ \\
\hline & 7 & 3,2 & 4,3 & 4,4 & 5,5 & $6,6, \mathrm{E}$ & $\ldots$ & $\ldots$ \\
\hline & 8 & 2,2 & 4,3 & 4,4 & 6,5 & 6,6 & $7,7, \mathrm{E}$ & $\ldots$ \\
\hline & 9 & 4,2 & $\overline{3,3}$ & 5,4 & $\overline{6,5}$ & 6,6 & 7,7 & $8,8, \mathrm{E}$ \\
\hline
\end{tabular}

state (and the Jacobian) of the system is required during the transformation. That is, direct attractor selection/targeting is possible-via the transformation surfaces summarized in Table I-without the application of a feedback. This is the main advantage of the ADT method: it enables attractor selection without sampling of the trajectory (or the Jacobian) of the solution and without the application of feedback.

The disadvantage of the ADT technique-being a feedforward control method-is that knowledge about the details of the bifurcation structure of the system is required. Otherwise, the proper route of the transformation of the periodic orbits with the temporary dualfrequency driving is not known. The "remedy" for this problem can be the application of an intermediate control quantity, for instance, the spectra of the emitted pressure in the case of bubbles. ${ }^{82,83}$ The peaks at the subharmonics in the spectra might help to properly tune the driving amplitudes during a transformation. Moreover, if the basin of attraction of the targeted orbit is small, the tuning of the pressure amplitudes $P_{A 1}$ and $P_{A 2}$ must be sufficiently slow. However, the discussion of this issue is beyond the scope of the present study. For a more detailed comparison of the advantages/disadvantages of the proposed ADT technique with methods of control of multi-stability, the interested reader is referred to our previous work. $^{23}$

As the ADT method is demonstrated only for a specific system (the Keller-Miksis equation), it is important to generalize the technique to other oscillators/models in the future. As a first step, examining other oscillators such as the Duffing, ${ }^{84-90}$ Toda, ${ }^{91-94}$ 

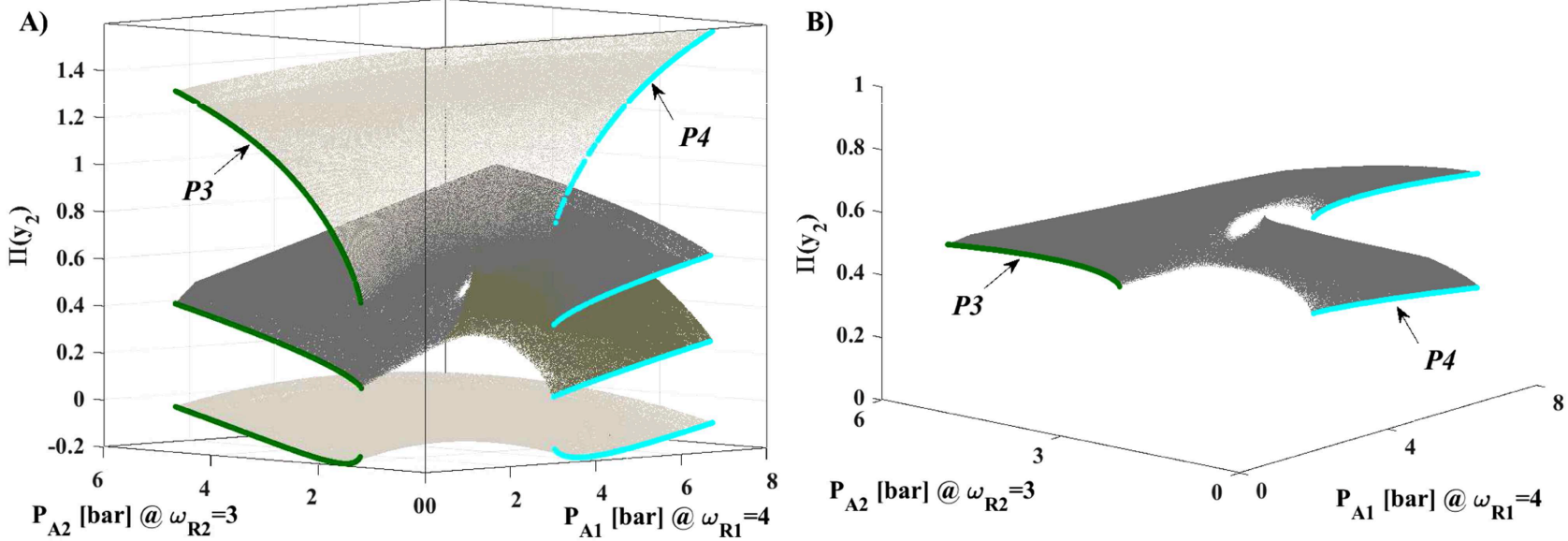

FIG. 8. Example of a frequency combination having branches with full connectivity. Panel (b) shows the middle surface presented in panel (a) from a different perspective for better visibility.

Morse, ${ }^{95,96}$ or van der Pol $^{97}$ equations will be an important step toward the generalization. Furthermore, testing the ADT technique on a large number of diffusively ${ }^{98-102}$ or globally ${ }^{103-107}$ coupled identical systems can have great significance as they can exhibit extreme multi-stability ${ }^{108}$ (the presence of infinitely many co-existing stable states), and they have practical relevance in many scientific areas. For instance, with the proposed method, it might be possible that in a diffusively coupled model, the rhythmicity can be restored. ${ }^{109}$ In acoustic cavitation and sonochemistry, ${ }^{110-112}$ the investigation of a bubble cluster ${ }^{113-116}$ (an ensemble of single bubbles coupled globally) can provide valuable information on how to drive the system into a chemically highly active state. As a final example, by driving an ultrasound contrast agent into higher-order subharmonics, the resolution of ultrasound imaging can potentially be increased improving the existing techniques in diagnostic ultrasound. ${ }^{117,118}$

Finally, it is worth highlighting that any application inherently using dual-frequency excitation for purposes other than controlling multi-stability and attractor targeting can still benefit (at least indirectly) from the detailed numerical results presented in this study. Without giving an exhaustive list, some of these applications are dual-frequency driven sonochemistry, ${ }^{110,119-122}$ stability analysis of traveling beams, ${ }^{123,124}$ or laser-driven dissociation of molecules. ${ }^{125}$

\section{SUMMARY}

A feedforward technique is presented that is capable of driving a periodically driven non-linear system between periodic orbits of subharmonic resonances: the Attractor Domain Targeting technique. This approach is demonstrated with the Keller-Miksis equation describing the evolution of the radial dynamics of a spherical gas bubble placed in an infinite domain of liquid. The basis of the method is the temporary addition of a second harmonic component to the driving. Results have shown that with the proper selection of the frequency pair and with the proper tuning of the amplitudes of the dual-frequency driving, continuous transformations exist between any pair of periodic orbits of subharmonic resonances. The technique was numerically tested on attractors with periods up to period 9. The frequency ratio must be equal to the ratio of the periods of the periodic orbits being transformed. As special cases, transformations have been found between period-1 orbits (originating from the equilibrium state of the system in the absence of external forcing) and attractors having a period higher than or equal to 5 ; see the items marked by $E$ in Table I.

\section{ACKNOWLEDGMENTS}

This paper was supported by the Alexander von Humboldt Foundation (Grant No. HUN 1162727 HFST-E), by the János Bolyai Research Scholarship of the Hungarian Academy of Sciences, by the Deutsche Forschungsgemeinschaft (DFG) (Grant No. ME 1645/71), and by the Higher Education Excellence Program of the Ministry of Human Capacities in the frame of Water Science \& Disaster Prevention research area of the Budapest University of Technology and Economics (No. BME FIKP-VÍZ).

\section{APPENDIX: THE DIMENSIONLESS FORM OF THE KELLER-MIKSIS EQUATION}

The Keller-Miksis equation, Eqs. (1)-(4), can be rewritten in a dimensionless form as follows:

$$
\dot{y}_{1}=y_{2} \text {, }
$$

$$
\dot{y}_{2}=\frac{N_{\mathrm{KM}}}{D_{\mathrm{KM}}},
$$


where the numerator, $N_{\mathrm{KM}}$, and the denominator, $D_{\mathrm{KM}}$, are

$$
\begin{aligned}
N_{\mathrm{KM}}= & \left(C_{0}+C_{1} y_{2}\right)\left(\frac{1}{y_{1}}\right)^{C_{10}}-C_{2}\left(1+C_{9} y_{2}\right)-C_{3} \frac{1}{y_{1}}-C_{4} \frac{y_{2}}{y_{1}} \\
& -\left(1-C_{9} \frac{y_{2}}{3}\right) \frac{3}{2} y_{2}^{2}-\left(C_{5} \sin (2 \pi \tau)\right. \\
& \left.+C_{6} \sin \left(2 \pi C_{11} \tau+C_{12}\right)\right)\left(1+C_{9} y_{2}\right) \\
& -y_{1}\left(C_{7} \cos (2 \pi \tau)+C_{8} \cos \left(2 \pi C_{11} \tau+C_{12}\right)\right)
\end{aligned}
$$

and

$$
D_{\mathrm{KM}}=y_{1}-C_{9} y_{1} y_{2}+C_{4} C_{9} \text {, }
$$

respectively.

The coefficients of the system are summarized as follows:

$$
\begin{gathered}
C_{0}=\frac{1}{\rho_{L}}\left(P_{\infty}-p_{V}+\frac{2 \sigma}{R_{E}}\right)\left(\frac{2 \pi}{R_{E} \omega_{1}}\right)^{2}, \\
C_{1}=\frac{1-3 \gamma}{\rho_{L} c_{L}}\left(P_{\infty}-p_{V}+\frac{2 \sigma}{R_{E}}\right) \frac{2 \pi}{R_{E} \omega_{1}} \\
C_{2}=\frac{P_{\infty}-p_{V}}{\rho_{L}}\left(\frac{2 \pi}{R_{E} \omega_{1}}\right)^{2}, \\
C_{3}=\frac{2 \sigma}{\rho_{L} R_{E}}\left(\frac{2 \pi}{R_{E} \omega_{1}}\right)^{2}, \\
C_{4}=\frac{4 \mu_{L}}{\rho_{L} R_{E}^{2}} \frac{2 \pi}{\omega_{1}}, \\
C_{5}=\frac{P_{A 1}}{\rho_{L}}\left(\frac{2 \pi}{R_{E} \omega_{1}}\right)^{2}, \\
C_{6}=\frac{P_{A 2}}{\rho_{L}}\left(\frac{2 \pi}{R_{E} \omega_{1}}\right)^{2}, \\
C_{7}=R_{E} \frac{\omega_{1} P_{A 1}}{\rho_{L} c_{L}}\left(\frac{2 \pi}{R_{E} \omega_{1}}\right)^{2}, \\
C_{8}=R_{E} \frac{\omega_{1} P_{A 2}}{\rho_{L} c_{L}}\left(\frac{2 \pi}{R_{E} \omega_{1}}\right)^{2}, \\
C_{9}=\frac{R_{E} \omega_{1}}{2 \pi c_{L}}, \\
C_{11}=\frac{\omega_{2}}{\omega_{1}}, \\
C_{12}=\theta \\
{ }_{1}
\end{gathered}
$$

\section{DATA AVAILABILITY}

The data that support the findings of this study are available from the corresponding author upon reasonable request.

\section{REFERENCES}

1J. G. Freire, A. Calderón-Cárdenas, H. Varela, and J. A. C. Gallas, "Phase diagrams and dynamical evolution of the triple-pathway electro-oxidation of formic acid on platinum," Phys. Chem. Chem. Phys. 22, 1078-1091 (2020).

${ }^{2}$ R. Varga, K. Klapcsik, and F. Hegedüs, "Route to shrimps: Dissipation driven formation of shrimp-shaped domains," Chaos Soliton Fractals 130, 109424 (2020).

${ }^{3}$ J. A. de Oliveira, L. T. Montero, D. R. da Costa, J. A. Méndez-Bermúdez, R. O. Medrano-T, and E. D. Leonel, "An investigation of the parameter space for a family of dissipative mappings," Chaos 29, 053114 (2019).

${ }^{4}$ D. W. C. Marcondes, G. F. Comassetto, B. G. Pedro, J. C. C. Vieira, A. Hoff, F. Prebianca, C. Manchein, and H. A. Albuquerque, "Extensive numerical study and circuitry implementation of the Watt governor model," Int. J. Bifurcat. Chaos 27, 1750175 (2017).

${ }^{5}$ A. C. C. Horstmann, H. A. Albuquerque, and C. Manchein, "The effect of temperature on generic stable periodic structures in the parameter space of dissipative relativistic standard map," Eur. Phys. J. B 90, 96 (2017).

${ }^{6}$ R. Rocha and R. O. Medrano-T, "Stability analysis and mapping of multiple dynamics of Chua's circuit in full four-parameter spaces," Int. J. Bifurcat. Chaos 25, 1530037 (2015).

${ }^{7}$ J. A. C. Gallas, "Periodic oscillations of the forced Brusselator," Mod. Phys. Lett. B 29, 1530018 (2015).

${ }^{8}$ A. Celestino, C. Manchein, H. A. Albuquerque, and M. W. Beims, "Stable structures in parameter space and optimal ratchet transport," Commun. Nonlinear Sci. Numer. Simul. 19, 139-149 (2014).

${ }^{9}$ S. L. T. de Souza, A. A. Lima, I. L. Caldas, R. O. Medrano-T, and Z. O. GuimaråesFilho, "Self-similarities of periodic structures for a discrete model of a two-gene system," Phys. Lett. A 376, 1290-1294 (2012).

${ }^{10} \mathrm{~J}$. G. Freire and J. A. C. Gallas, "Stern-Brocot trees in cascades of mixedmode oscillations and canards in the extended Bonhoeffer-van der Pol and the Fitzhugh-Nagumo models of excitable systems," Phys. Lett. A 375, 1097-1103 (2011).

${ }^{11}$ J. G. Freire and J. A. C. Gallas, "Stern-Brocot trees in the periodicity of mixedmode oscillations," Phys. Chem. Chem. Phys. 13, 12191-12198 (2011).

${ }^{12}$ A. Celestino, C. Manchein, H. A. Albuquerque, and M. W. Beims, "Ratchet transport and periodic structures in parameter space," Phys. Rev. Lett. 106, 234101 (2011).

${ }^{13}$ M. A. Nascimento, J. A. C. Gallas, and H. Varela, "Self-organized distribution of periodicity and chaos in an electrochemical oscillator," Phys. Chem. Chem. Phys. 13, 441-446 (2011).

${ }^{14}$ S. Luther, F. H. Fenton, B. G. Kornreich, A. Squires, P. Bittihn, D. Hornung, M. Zabel, J. Flanders, A. Gladuli, L. Campoy, E. M. Cherry, G. Luther, G. Hasenfuss, V. I. Krinsky, A. Pumir, R. F. Gilmour, and E. Bodenschatz, "Low-energy control of electrical turbulence in the heart," Nature 475, 235-239 (2011).

${ }^{15}$ A. Karma, "Physics of cardiac arrhythmogenesis," Annu. Rev. Condens. Matter Phys. 4, 313-337 (2013).

${ }^{16} \mathrm{~J}$. Tomek, B. Rodriguez, G. Bub, and J. Heijman, " $\beta$-Adrenergic receptor stimulation inhibits proarrhythmic alternans in postinfarction border zone cardiomyocytes: A computational analysis," Am. J. Physiol. Heart Circ. Physiol. 313, H338-H353 (2017).

${ }^{17}$ E. Boccia, S. Luther, and U. Parlitz, "Modelling far field pacing for terminating spiral waves pinned to ischaemic heterogeneities in cardiac tissue," Philos. Trans. R. Soc. A 375, 20160289 (2017).

${ }^{18}$ A. E. Motter, S. A. Myers, M. Anghel, and T. Nishikawa, "Spontaneous synchrony in power-grid networks,” Nat. Phys. 9, 191-197 (2013).

${ }^{19}$ E. S. Medeiros, R. O. Medrano-T, I. L. Caldas, T. Tél, and U. Feudel, "State-dependent vulnerability of synchronization," Phys. Rev. E 100, 052201 (2019).

${ }^{20}$ X. Li, T. Qiu, S. Boccaletti, I. Sendiña-Nadal, Z. Liu, and S. Guan, "Synchronization clusters emerge as the result of a global coupling among classical phase oscillators," New J. Phys. 21, 053002 (2019).

${ }^{21}$ E. S. Medeiros, R. O. Medrano-T, I. L. Caldas, and U. Feudel, "Boundaries of synchronization in oscillator networks," Phys. Rev. E 98, 030201 (2018).

${ }^{22}$ N. Inaba, H. Ito, K. Shimizu, and H. Hikawa, "Complete mixed-mode oscillation synchronization in weakly coupled nonautonomous Bonhoeffer-van der Pol oscillators," Prog. Theor. Exp. Phys. 2018, 063 A01. 
${ }^{23}$ F. Hegedűs, W. Lauterborn, U. Parlitz, and R. Mettin, "Non-feedback technique to directly control multistability in nonlinear oscillators by dual-frequency driving," Nonlinear Dyn. 94, 273-293 (2018).

${ }^{24}$ A. N. Pisarchik and U. Feudel, "Control of multistability," Phys. Rep. 540, 167-218 (2014).

${ }^{25}$ A. N. Pisarchik and B. K. Goswami, "Annihilation of one of the coexisting attractors in a bistable system," Phys. Rev. Lett. 84, 1423-1426 (2000).

${ }^{26} \mathrm{~K}$. Kaneko, "Dominance of Milnor attractors and noise-induced selection in a multiattractor system," Phys. Rev. Lett. 78, 2736-2739 (1997).

${ }^{27}$ J. B. Keller and M. Miksis, “Bubble oscillations of large amplitude," J. Acoust. Soc. Am. 68, 628-633 (1980).

${ }^{28} \mathrm{Y}$. Zhang and Y. Zhang, "Chaotic oscillations of gas bubbles under dualfrequency acoustic excitation," Ultrason. Sonochem. 40, 151-157 (2018).

${ }^{29} \mathrm{H}$. Haghi, A. J. Sojahrood, and M. C. Kolios, "Collective nonlinear behavior of interacting polydisperse microbubble clusters," Ultrason. Sonochem. 58, 104708 (2019).

${ }^{30} \mathrm{~W}$. Lauterborn and T. Kurz, "Physics of bubble oscillations," Rep. Prog. Phys. 73, 106501 (2010).

${ }^{31}$ W. Lauterborn and U. Parlitz, "Methods of chaos physics and their application to acoustics," J. Acoust. Soc. Am. 84, 1975-1993 (1988).

${ }^{32}$ C. E. Brennen, Cavitation and Bubble Dynamics (Oxford University Press, New York, 1995).

${ }^{33}$ E. J. Doedel, B. E. Oldeman, A. R. Champneys, F. Dercole, T. F. Fairgrieve, Y. A. Kuznetsov, R. Paffenroth, B. Sandstede, X. Wang, and C. Zhang, AUTO-07P: Continuation and Bifurcation Software for Ordinary Differential Equations (Concordia University, Montreal, Canada, 2012).

${ }^{34}$ See www.gpuode.com for "Massively Parallel GPU-ODE Solver (MPGOS)" (2019).

${ }^{35} \mathrm{~F}$. Hegedüs, MPGOS: GPU Accelerated Integrator for Large Number of Independent Ordinary Differential Equation Systems (Budapest University of Technology and Economics, Budapest, Hungary, 2019).

${ }^{36}$ See https://github.com/ferenchegedus/massively-parallel-gpu-ode-solver for "Massively Parallel GPU-ODE Solver" (2019).

${ }^{37}$ L. Poon and C. Grebogi, "Controlling complexity," Phys. Rev. Lett. 75, 4023-4026 (1995).

${ }^{38} \mathrm{U}$. Feudel and C. Grebogi, "Multistability and the control of complexity," Chaos 7, 597-604 (1997).

${ }^{39}$ Y. C. Lai, "Driving trajectories to a desirable attractor by using small control," Phys. Lett. A 221, 375-383 (1996).

${ }^{40}$ E. E. N. Macau and C. Grebogi, "Driving trajectories in complex systems," Phys. Rev. E 59, 4062-4070 (1999).

${ }^{41}$ S. Gadaleta and G. Dangelmayr, "Learning to control a complex multistable system," Phys. Rev. E 63, 036217 (2001).

${ }^{42}$ T. Shinbrot, E. Ott, C. Grebogi, and J. A. Yorke, "Using chaos to direct trajectories to targets," Phys. Rev. Lett. 65, 3215-3218 (1990).

${ }^{43}$ Y. Jiang, "Trajectory selection in multistable systems using periodic drivings," Phys. Lett. A 264, 22-29 (1999).

${ }^{44} \mathrm{~K}$. Ikeda, "Multiple-valued stationary state and its instability of the transmitted light by a ring cavity system," Opt. Commun. 30, 257-261 (1979).

${ }^{45} \mathrm{~K}$. Ikeda and K. Matsumoto, "High-dimensional chaotic behavior in systems with time-delayed feedback," Physica D 29, 223-235 (1987).

${ }^{46} \mathrm{~K}$. Pyragas, "Continuous control of chaos by self-controlling feedback," Phys. Lett. A 170, 421-428 (1992).

${ }^{47}$ B. E. Martínez-Zérega, A. N. Pisarchik, and L. S. Tsimring, "Using periodic modulation to control coexisting attractors induced by delayed feedback," Phys. Lett. A 318, 102-111 (2003).

${ }^{48}$ B. E. Martínez-Zérega and A. N. Pisarchik, "Efficiency of the control of coexisting attractors by harmonic modulation applied in different ways," Phys. Lett. A 340, 212-219 (2005)

${ }^{49}$ A. N. Pisarchik, R. Meucci, and F. T. Arecchi, "Discrete homoclinic orbits in a laser with feedback," Phys. Rev. E 62, 8823-8825 (2000).

${ }^{50}$ A. N. Pisarchik, R. Meucci, and F. T. Arecchi, "Theoretical and experimental study of discrete behavior of Shilnikov chaos in a $\mathrm{CO}_{2}$ laser," Eur. Phys. J. D 13, 385-391 (2001).
${ }^{51}$ Y. Zhang, Y. Zhang, and S. Li, "Combination and simultaneous resonances of gas bubbles oscillating in liquids under dual-frequency acoustic excitation," Ultrason. Sonochem. 35, 431-439 (2017).

${ }^{52} \mathrm{Y}$. Zhang, Y. Zhang, and S. Li, "The secondary Bjerknes force between two gas bubbles under dual-frequency acoustic excitation," Ultrason. Sonochem. 29, 129-145 (2016).

${ }^{53}$ A. J. Sojahrood, H. Haghi, Q. Li, T. M. Porter, R. Karshafian, and M. C. Kolios, "Nonlinear power loss in the oscillations of coated and uncoated bubbles: Role of thermal, radiation and encapsulating shell damping at various excitation pressures," Ultrason. Sonochem. 66, 105070 (2020).

${ }^{54}$ A. J. Sojahrood, O. Falou, R. Earl, R. Karshafian, and M. C. Kolios, "Influence of the pressure-dependent resonance frequency on the bifurcation structure and backscattered pressure of ultrasound contrast agents: A numerical investigation," Nonlinear Dyn. 80, 889-904 (2015).

${ }^{55} \mathrm{~K}$. Yasui, T. Tuziuti, J. Lee, T. Kozuka, A. Towata, and Y. Iida, "The range of ambient radius for an active bubble in sonoluminescence and sonochemical reactions," J. Chem. Phys. 128, 184705 (2008).

${ }^{56} \mathrm{~K}$. Yasui, T. Tuziuti, T. Kozuka, A. Towata, and Y. Iida, "Relationship between the bubble temperature and main oxidant created inside an air bubble under ultrasound," J. Chem. Phys. 127, 154502 (2007).

${ }^{57} \mathrm{~K}$. Yasui, T. Tuziuti, and Y. Iida, "Optimum bubble temperature for the sonochemical production of oxidants," Ultrasonics 42, 579-584 (2004).

${ }^{58} \mathrm{Y}$. Hao and A. Prosperetti, "The dynamics of vapor bubbles in acoustic pressure fields," Phys. Fluids 11, 2008-2019 (1999).

${ }^{59} \mathrm{~L}$. Stricker and D. Lohse, "Radical production inside an acoustically driven microbubble," Ultrason. Sonochem. 21, 336-345 (2014).

${ }^{60}$ R. Mettin, I. Akhatov, U. Parlitz, C. D. Ohl, and W. Lauterborn, "Bjerknes forces between small cavitation bubbles in a strong acoustic field," Phys. Rev. E 56, 2924-2931 (1997).

${ }^{61}$ R. Mettin, S. Luther, C.-D. Ohl, and W. Lauterborn, "Acoustic cavitation structures and simulations by a particle model," Ultrason. Sonochem. 6, 25-29 (1999).

${ }^{62} \mathrm{E}$. Li, "Chromatin modification and epigenetic reprogramming in mammalian development,” Nat. Rev. Genet. 3, 662-673 (2002).

${ }^{63}$ V. N. Chizhevsky, "Coexisting attractors in a $\mathrm{CO}_{2}$ laser with modulated losses," J. Opt. B Quantum Semiclassical Opt. 2, 711-717 (2000).

${ }^{64} \mathrm{~V}$. N. Chizhevsky, R. Vilaseca, and R. Corbalán, "Experimental switchings in bistability domains induced by resonant perturbations," Int. J. Bifurcat. Chaos $\mathbf{8}$, 1777-1782 (1998).

${ }^{65}$ V. N. Chizhevsky, R. Corbalán, and A. N. Pisarchik, "Attractor splitting induced by resonant perturbations," Phys. Rev. E 56, 1580-1584 (1997).

${ }^{66}$ V. N. Chizhevsky, E. V. Grigorieva, and S. A. Kashchenko, "Optimal timing for targeting periodic orbits in a loss-driven $\mathrm{CO}_{2}$ laser," Opt. Commun. 133, 189-195 (1997).

${ }^{67}$ V. N. Chizhevsky and P. Glorieux, "Targeting unstable periodic orbits," Phys. Rev. E 51, R2701-R2704 (1995).

${ }^{68} \mathrm{~V}$. N. Chizhevsky and S. I. Turovets, "Periodically loss-modulated $\mathrm{CO}_{2}$ laser as an optical amplitude and phase multitrigger," Phys. Rev. A 50, 1840-1843 (1994).

${ }^{69} \mathrm{~K}$. Kaneko, "Clustering, coding, switching, hierarchical ordering, and control in a network of chaotic elements," Physica D 41, 137-172 (1990).

${ }^{70} \mathrm{~V}$. N. Chizhevsky and S. I. Turovets, "Small signal amplification and classical squeezing near period-doubling bifurcations in a modulated $\mathrm{CO}_{2}$-laser," Opt. Commun. 102, 175-182 (1993).

${ }^{71} \mathrm{~K}$. Kaneko, "Chaotic but regular posi-nega switch among coded attractors by cluster-size variation,” Phys. Rev. Lett. 63, 219-223 (1989).

${ }^{72}$ B. F. Kuntsevich, A. N. Pisarchik, V. N. Chizhevskii, and V. V. Churakov, "Amplitude modulation of the radiation of a $\mathrm{CO}_{2}$-laser by optically controlled absorption in semiconductors," J. Appl. Spectrosc. 38, 107-112 (1983).

${ }^{73}$ L. M. Pecora and T. L. Carroll, "Pseudoperiodic driving: Eliminating multiple domains of attraction using chaos," Phys. Rev. Lett. 67, 945-948 (1991).

${ }^{74}$ T. L. Carroll and L. M. Pecora, "Using chaos to keep period-multiplied systems in phase," Phys. Rev. E 48, 2426-2436 (1993).

${ }^{75}$ A. N. Pisarchik, "Controlling the multistability of nonlinear systems with coexisting attractors,” Phys. Rev. E 64, 046203 (2001). 
${ }^{76}$ B. K. Goswami, S. Euzzor, K. Al Naimee, A. Geltrude, R. Meucci, and F. T. Arecchi, "Control of stochastic multistable systems: Experimental demonstration," Phys. Rev. E 80, 016211 (2009).

${ }^{77}$ A. N. Pisarchik, Y. O. Barmenkov, and A. V. Kir'yanov, "Experimental demonstration of attractor annihilation in a multistable fiber laser," Phys. Rev. E 68, 066211 (2003).

${ }^{78}$ A. N. Pisarchik and R. Corbalán, "Shift of attractor boundaries in a system with a slow harmonic parameter perturbation," Physica D 150, 14-24 (2001).

${ }^{79}$ A. N. Pisarchik and B. F. Kuntsevich, "Control of multistability in a directly modulated diode laser,” IEEE J. Quantum Electron. 38, 1594-1598 (2002).

${ }^{80} \mathrm{~A}$. N. Pisarchik and R. Jaimes-Reategui, "Control of basins of attraction in a multistable fiber laser,” Phys. Lett. A 374, 228-234 (2009).

${ }^{81}$ R. Mettin, and T. Kurz, "Optimized periodic control of chaotic systems," Phys. Lett. A 206(5-6), 331-339 (1995).

${ }^{82}$ W. Lauterborn and J. Holzfuss, “Acoustic chaos," Int. J. Bifurcat. Chaos 1, 13-26 (1991).

${ }^{83}$ W. Lauterborn and E. Cramer, "Subharmonic route to chaos observed in acoustics,” Phys. Rev. Lett. 47, 1445-1448 (1981).

${ }^{84}$ C. Bonatto, J. A. C. Gallas, and Y. Ueda, "Chaotic phase similarities and recurrences in a damped-driven Duffing oscillator," Phys. Rev. E 77, 026217 (2008).

${ }^{85} \mathrm{~V}$. Englisch and W. Lauterborn, "Regular window structure of a double-well Duffing oscillator," Phys. Rev. A 44, 916-924 (1991).

${ }^{86}$ R. Gilmore and J. W. L. McCallum, "Structure in the bifurcation diagram of the Duffing oscillator,” Phys. Rev. E 51, 935-956 (1995).

${ }^{87}$ Y. H. Kao, J. C. Huang, and Y. S. Gou, "Persistent properties of crises in a Duffing oscillator,” Phys. Rev. A 35, 5228-5232 (1987).

${ }^{88} \mathrm{~J}$. Kozłowski, U. Parlitz, and W. Lauterborn, "Bifurcation analysis of two coupled periodically driven Duffing oscillators,” Phys. Rev. E 51, 1861-1867 (1995).

${ }^{89} \mathrm{U}$. Parlitz and W. Lauterborn, "Superstructure in the bifurcation set of the Duffing equation $\ddot{x}+d \dot{x}+x+x^{3}=f \cos (\omega t)$," Phys. Lett. A 107, 351-355 (1985).

${ }^{90}$ C. S. Wang, Y. H. Kao, J. C. Huang, and Y. S. Gou, "Potential dependence of the bifurcation structure in generalized Duffing oscillators," Phys. Rev. A 45, 3471-3485 (1992).

${ }^{91}$ T. Kurz and W. Lauterborn, "Bifurcation structure of the Toda oscillator," Phys. Rev. A 37, 1029-1031 (1988).

${ }^{92} \mathrm{~B}$. K. Goswami, "The interaction between period 1 and period 2 branches and the recurrence of the bifurcation structures in the periodically forced laser rate equations," Opt. Commun. 122, 189-199 (1996).

${ }^{93}$ B. K. Goswami, "Self-similarity in the bifurcation structure involving period tripling, and a suggested generalization to period n-tupling," Phys. Lett. A 245, 97-109 (1998)

${ }^{94}$ B. K. Goswami, "Flip-flop between soft-spring and hard-spring bistabilities in the approximated Toda oscillator analysis," Pramana 77, 987-1005 (2011).

${ }^{95} \mathrm{~W}$. Knop and W. Lauterborn, "Bifurcation structure of the classical Morse oscillator,” J. Chem. Phys. 93, 3950-3957 (1990).

${ }^{96}$ C. Scheffczyk, U. Parlitz, T. Kurz, W. Knop, and W. Lauterborn, "Comparison of bifurcation structures of driven dissipative nonlinear oscillators," Phys. Rev. A 43, 6495-6502 (1991).

${ }^{97}$ R. Mettin, U. Parlitz, and W. Lauterborn, "Bifurcation structure of the driven van der Pol oscillator,” Int. J. Bifurcat. Chaos 03, 1529-1555 (1993).

${ }^{98} \mathrm{P}$. Wieland, J. Wu, and F. Allgöwer, "On synchronous steady states and internal models of diffusively coupled systems," IEEE Trans. Automat. Contr. 58, 2591-2602 (2013).

${ }^{99}$ C. R. Hens, O. I. Olusola, P. Pal, and S. K. Dana, "Oscillation death in diffusively coupled oscillators by local repulsive link,” Phys. Rev. E 88, 034902 (2013).

${ }^{100}$ W. Xia and M. Cao, "Clustering in diffusively coupled networks," Automatica 47, 2395-2405 (2011).

${ }^{101} \mathrm{~J}$. Halatek and E. Frey, "Rethinking pattern formation in reaction-diffusion systems," Nat. Phys. 14, 507-514 (2018).

${ }^{102}$ J. Tomek, G. Hao, M. Tomková, A. Lewis, C. Carr, D. J. Paterson, B. Rodriguez, G. Bub, and N. Herring, " $\beta$-Adrenergic receptor stimulation and alternans in the border zone of a healed infarct: An ex vivo study and computational investigation of arrhythmogenesis," Front. Physiol. 10, 350 (2019).
${ }^{103}$ V. K. Chandrasekar, R. Gopal, A. Venkatesan, and M. Lakshmanan, "Mechanism for intensity-induced chimera states in globally coupled oscillators," Phys. Rev. E 90, 062913 (2014).

${ }^{104}$ A. Yeldesbay, A. Pikovsky, and M. Rosenblum, "Chimeralike states in an ensemble of globally coupled oscillators,” Phys. Rev. Lett. 112, 144103 (2014).

${ }^{105} \mathrm{~S}$. Eydam and M. Wolfrum, "Mode locking in systems of globally coupled phase oscillators," Phys. Rev. E 96, 052205 (2017).

${ }^{106}$ A. V. Cano and M. G. Cosenza, "Asymmetric cluster and chimera dynamics in globally coupled systems,” Chaos 28, 113119 (2018).

${ }^{107}$ J. D. Hart, K. Bansal, T. E. Murphy, and R. Roy, "Experimental observation of chimera and cluster states in a minimal globally coupled network," Chaos 26, 094801 (2016).

${ }^{108}$ C. R. Hens, S. K. Dana, and U. Feudel, "Extreme multistability: Attractor manipulation and robustness," Chaos 25, 053112 (2015).

${ }^{109}$ W. Zou, D. V. Senthilkumar, R. Nagao, I. Z. Kiss, Y. Tang, A. Koseska, J. Duan, and J. Kurths, "Restoration of rhythmicity in diffusively coupled dynamical networks,” Nat. Commun. 6, 7709 (2015).

110 A. Brotchie, F. Grieser, and M. Ashokkumar, "Sonochemistry and sonoluminescence under dual-frequency ultrasound irradiation in the presence of water-soluble solutes," J. Phys. Chem. C 112, 10247-10250 (2008).

${ }^{111}$ W. Lauterborn and C.-D. Ohl, "Acoustic cavitation and multi bubble sonoluminescence," in Sonochemistry and Sonoluminescence, edited by L. A. Crum, T. J. Mason, J. L. Reisse, and K. S. Suslick (Springer Netherlands, Dordrecht, 1999), pp. 97-104.

${ }^{112}$ R. Mettin, C. Cairós, and A. Troia, "Sonochemistry and bubble dynamics," Ultrason. Sonochem. 25, 24-30 (2015).

${ }^{113}$ S. Behnia, H. Zahir, M. Yahyavi, A. Barzegar, and F. Mobadersani, "Observations on the dynamics of bubble cluster in an ultrasonic field," Nonlinear Dyn. 72, 561-574 (2013).

${ }^{114} \mathrm{C} . \mathrm{H}$. Wang and J. C. Cheng, "Interaction of a bubble and a bubble cluster in an ultrasonic field," Chin. Phys. B 22, 014304 (2013).

${ }^{115}$ K. Yasui, T. Tuziuti, J. Lee, T. Kozuka, A. Towata, and Y. Iida, "Numerical simulations of acoustic cavitation noise with the temporal fluctuation in the number of bubbles," Ultrason. Sonochem. 17, 460-472 (2010).

${ }^{116}$ S. Luther, M. Sushchik, U. Parlitz, I. Akhatov, and W. Lauterborn, "Is cavitation noise governed by a low-dimensional chaotic attractor?," AIP Conf. Proc. 524, 355-358 (2000).

${ }^{117}$ A. de Leon, R. Perera, C. Hernandez, M. Cooley, O. Jung, S. Jeganathan, E. Abenojar, G. Fishbein, A. J. Sojahrood, C. C. Emerson, P. L. Stewart, M. C. Kolios, and A. A. Exner, "Contrast enhanced ultrasound imaging by nature-inspired ultrastable echogenic nanobubbles," Nanoscale 11, 15647-15658 (2019).

118 A. J. Sojahrood and M. C. Kolios, "Classification of the nonlinear dynamics and bifurcation structure of ultrasound contrast agents excited at higher multiples of their resonance frequency," Phys. Lett. A 376, 2222-2229 (2012).

${ }^{119}$ S. Behnia, A. J. Sojahrood, W. Soltanpoor, and O. Jahanbakhsh, "Suppressing chaotic oscillations of a spherical cavitation bubble through applying a periodic perturbation," Ultrason. Sonochem. 16, 502-511 (2009).

${ }^{120}$ S. D. Sokka, T. P. Gauthier, and K. Hynynen, "Theoretical and experimental validation of a dual-frequency excitation method for spatial control of cavitation," Phys. Med. Biol. 50, 2167-2179 (2005).

${ }^{121}$ N. B. Waldo and C. D. Vecitis, "Combined effects of phase-shift and power distribution on efficiency of dual-high-frequency sonochemistry," Ultrason. Sonochem. 41, 100-108 (2018).

${ }^{122}$ F. Hegedűs, K. Klapcsik, W. Lauterborn, U. Parlitz, and R. Mettin, “GPU accelerated study of a dual-frequency driven single bubble in a 6-dimensional parameter space: The active cavitation threshold," Ultrason. Sonochem. 67, 105067 (2020).

${ }^{123}$ B. Sahoo, L. N. Panda, and G. Pohit, "Two-frequency parametric excitation and internal resonance of a moving viscoelastic beam," Nonlinear Dyn. 82, 1721-1742 (2015).

${ }^{124}$ B. Sahoo, L. N. Panda, and G. Pohit, "Combination, principal parametric and internal resonances of an accelerating beam under two frequency parametric excitation,” Int. J. Nonlinear Mech. 78, 35-44 (2016).

${ }^{125}$ S. Huang, C. Chandre, and T. Uzer, "Bifurcations as dissociation mechanism in bichromatically driven diatomic molecules,” J. Chem. Phys. 128, 174105 (2008). 